\title{
Heme arginate improves reperfusion patterns after ischemia: a randomized, placebo-controlled trial in healthy male subjects
}

Martin Andreas ${ }^{1,2}$, Albrecht Ingo Schmid ${ }^{1,3}$, Daniel Doberer ${ }^{1}$, Kiril Schewzow $^{3}$, Stefan Weisshaar ${ }^{1}$, Georg Heinze $^{4}$, Martin Bilban ${ }^{5}$, Ewald Moser ${ }^{3}$ and Michael Wolzt ${ }^{1 *}$

\begin{abstract}
Background: Heme arginate can induce heme oxygenase-1 to protect tissue against ischemia-reperfusion injury. Blood oxygen level dependent (BOLD) functional magnetic resonance imaging measures changes in tissue oxygenation with a high spatial and temporal resolution. BOLD imaging was applied to test the effect of heme arginate on experimental ischemia reperfusion injury in the calf muscles.
\end{abstract}

Methods: A two period, controlled, observer blinded, crossover trial was performed in 12 healthy male subjects. Heme arginate (1 mg/kg body weight) or placebo were infused $24 \mathrm{~h}$ prior to a $20 \mathrm{~min}$ leg ischemia induced by a thigh cuff. 3 Tesla BOLD-imaging of the calf was performed and signal time courses from soleus, gastrocnemius and tibialis anterior muscle were available from 11 participants for technical reasons.

Results: Peak reactive hyperemia signal of the musculature was significantly increased and occurred earlier after heme arginate compared to placebo $(106.2 \pm 0.6 \%$ at $175 \pm 16 \mathrm{~s}$ vs. $104.5 \pm 0.6 \%$ at $221 \pm 19 \mathrm{~s} ; \mathrm{p}=0.025$ for peak reperfusion and $p=0.012$ for time to peak).

Conclusions: A single high dose of heme arginate improves reperfusion patterns during ischemia reperfusion injury in humans. BOLD sensitive, functional MRI is applicable for the assessment of experimental ischemia reperfusion injury in skeletal muscle.

\section{Trial registration}

ClinicalTrials: NCT01461512

EudraCT: 2008-006967-35

\section{Background}

Rapid reperfusion is essential for ischemic muscle. However, reperfusion itself can result in additional damage to ischemic tissue $[1,2]$. One of the key mechanisms is the rise of free oxygen radicals, thereby exceeding the cellular antioxidant capacity and damaging cellular proteins and membranes [3]. In a second step, neutrophil activation and tissue invasion is part of the deleterious cascade of ischemia-reperfusion injury (IRI) [4].

Several strategies to alleviate IRI have been tested in various experimental settings, including mechanical preconditioning and postconditioning, pharmacologic

\footnotetext{
*Correspondence: michael.wolzt@meduniwien.ac.at

'Department of Clinical Pharmacology, Medical University of Vienna, Vienna, Austria

Full list of author information is available at the end of the article
}

preconditioning, administration of antioxidant substances and treatment with endothelin receptor antagonists [5-10]. As a new therapeutic approach in humans, the induction of the enzyme heme oxygenase $1(\mathrm{HO}-1)$ may be employed to mitigate IRI $[10,11]$. $\mathrm{HO}-1$ is the ratelimiting enzyme for the degradation of heme $b$ [12]. Thereby, it produces biliverdin, carbon monoxide (CO) and iron. Recently, the role of $\mathrm{HO}-1$ as a protective enzyme was proposed due to its anti-inflammatory, antioxidant, anti-apoptotic and antiproliferative actions $[11,13]$. HO-1 is expressed in several organs including endothelial and smooth muscle cells in response to cellular stress conditions [14-16].

IRI may be attenuated by pharmacological HO-1 induction with heme arginate (HA) as shown in a rodent hemorrhagic shock model [11,17-19]. Previous data have

\section{Biomed Central}


confirmed dose-dependent induction of HO-1 mRNA and protein by HA in venous blood of healthy subjects [20,21].

In this therapeutic exploratory study, we aimed to evaluate the effects of HA on skeletal muscle IRI in healthy humans. Blood oxygen level dependent (BOLD) functional magnetic resonance imaging (fMRI) was used to measure alterations in tissue oxygenation with a high spatial and temporal resolution [22,23]. fMRI has previously been used to assess perfusion of the calf muscles in elderly people and patients with peripheral arterial occlusive disease after ischemia reperfusion experiments [24-26]. Further, we could measure an effect of ischemic preconditioning in healthy subjects on BOLD fMRI peak signal during reperfusion [27].

\section{Methods}

A two period placebo controlled, observer blinded, randomized crossover trial was performed in 12 healthy male subjects between 18 and 46 years (age [mean \pm standard deviation] $28 \pm 6$ years, body mass index 22.6 $\pm 2.1 \mathrm{~kg} / \mathrm{m}^{2}$ ). Functional MRI signals could not be analysed in one participant for technical reasons. Therefore, data are shown for 11 subjects. The study protocol was approved by the Ethics Committee of the Medical University of Vienna. All participants gave written informed consent prior to inclusion. One screening visit and two study periods with a washout time of at least 10 days in-between were scheduled for each participant. Physical examination and medical history, routine blood works including blood count, clinical chemistry, urine analysis and a 12-lead electrocardiogram were performed at the screening visit one week prior to the first study day. Subjects did not take any medication throughout the study and abstained from alcohol, stimulating beverages containing xanthine derivatives (tea, coffee) and heavy physical exercise 48 hours before drug infusion and fMRI. Subjects were fasted on study days. After the last treatment a final follow-up examination was performed.

A single dose of $1 \mathrm{mg} / \mathrm{kg}$ heme arginate or placebo (sodium chloride) was administered as an intravenous infusion in the alternate study periods. Heme arginate was diluted to $110 \mathrm{ml}$ with $0.9 \% \mathrm{NaCl}$. The infusion was administered within 15 minutes at an infusion rate of $440 \mathrm{ml} / \mathrm{h}$ using an infusomat (IP 85-2, Döring, Munich, Germany). A post-treatment infusion of $250 \mathrm{ml} 0.9 \%$ $\mathrm{NaCl}$ was administered to rinse perfusion lines.

Each study period consisted of three days. On the first day, placebo or heme arginate were administered. On the second day, the fMRI and ischemia reperfusion protocol was performed. On the third day, a blood draw for analysis of creatine kinase, lactate dehydrogenase, total protein, blood iron and $\mathrm{C}$ - reactive proteine as well as a physical examination were done.

Ischemia was administered to the right lower leg for 20 minutes using a cuff inflated to $200 \mathrm{mmHg}$ on the thigh (Figure 1b). The cuff was deflated to $30 \mathrm{mmHg}$ below systolic pressure for five minutes for slow reperfusion, mimicking impaired blood perfusion through the femoral artery. This setting has been validated previously [27]. A blood draw for $\mathrm{NO}_{2} / \mathrm{NO}_{3}$ analysis was performed a)

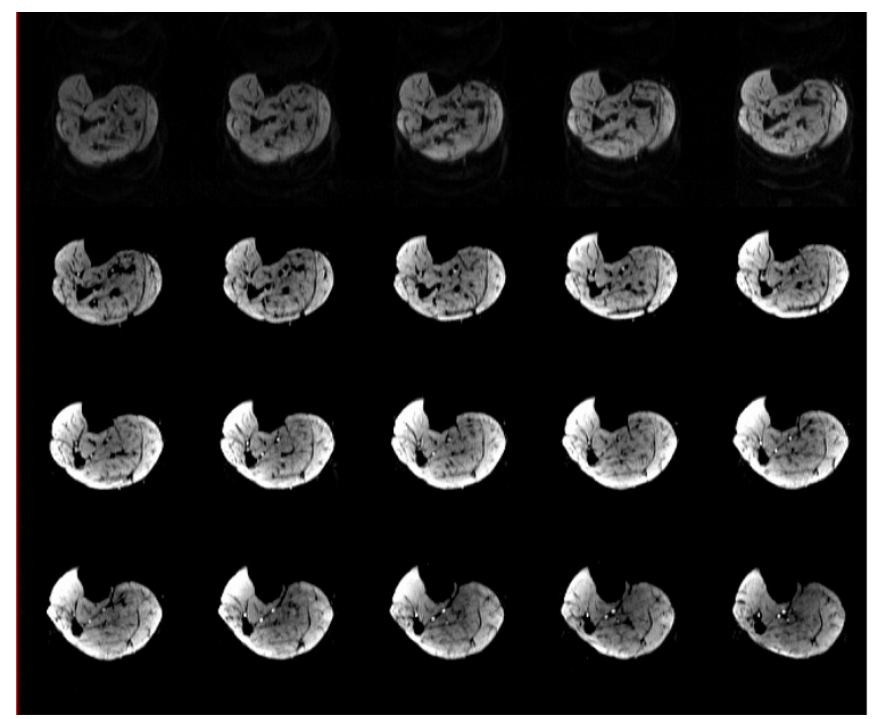

b) fMRI timeline

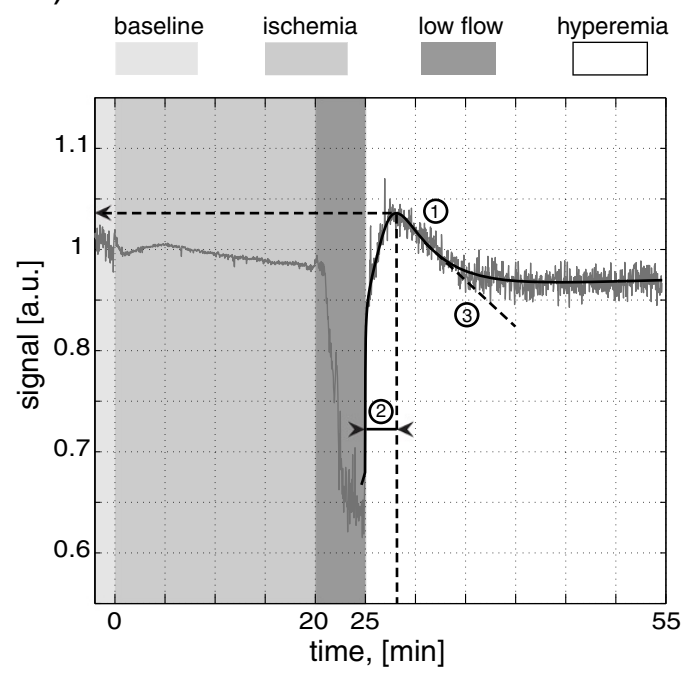

Figure 1 Experimental setup. a) EPI images for every $4^{\text {th }}$ minute during the experiment; b) fMRI timeline, a sample data set and description of parameters derived from fitted curves. Caption: Time course is showed in minutes; 1: peak BOLD signal; 2: time to peak; 3: tangent slope; a.u.: arbitrary units; min: minutes. 
before heme arginate infusion as well as prior to and 15 minutes after ischemia.

MR operators were blinded to the randomization sequence. A Siemens Tim Trio (Siemens Medical Solutions, Erlangen, Germany) was used for scanning. Anatomical images were acquired at the beginning of the MR examination. BOLD-imaging (EPI) had the following parameters: $128 \times 128$ matrix, twenty $5 \mathrm{~mm}$ axial slices, $1,42 \mathrm{~mm}$ in-plane resolution, $\mathrm{T}_{\mathrm{R}}=2 \mathrm{~s}, \mathrm{~T}_{\mathrm{E}}=28 \mathrm{~ms}$. 1700 EPI stacks were acquired every $T_{R}$. Imaging started two minutes before ischemia and continued for at least 25 minutes after reperfusion (Figure $1 \mathrm{a}+\mathrm{b}$ ). Regions of interest (ROI) were drawn around the soleus, gastrocnemius and tibialis anterior muscle for signal analysis. The BOLD signals in the ROIs as well as from all voxels were summed to achieve a single time course for each individual muscle and the complete calf respectively. The starting point of reperfusion was determined manually in all individual data sets.

To describe the BOLD response in calf muscle during reperfusion, the intensity time courses were fitted against a similar function as is used in DCE first pass perfusion measurements using Matlab (Mathworks, Natick, MA, USA) using the Curve Fitting Toolbox (Schewzow et al., submitted):

$$
f(t)=g(t)+s(t)+l(t)
$$

is the sum of gamma variate function $g(t)$

$$
g(t)=g_{0} \cdot\left(t-t_{0}\right)^{g_{l}} \cdot \mathrm{e}^{-g_{2}\left(t-t_{0}\right)}
$$

and a sigmoid function $s(t)$

$$
s(t)=s_{0} \cdot \frac{1}{1+\mathrm{e}^{-s_{I}\left(t-t_{0}-t_{I}\right)}}
$$

A linear function $l(t)$ was also added to take into account a possible drift of the signal during the experiment

$$
l(t)=l_{0}+l_{l}\left(t-t_{0}\right)
$$

The values of peak BOLD signal, time to peak and tangent slope were determined numerically from the fitted curves $\mathrm{f}(\mathrm{t})$ (Figure $1 \mathrm{~b})$.

The effect of heme arginate infusion on nitric oxide synthase was measured by $\mathrm{NO}_{2}$ and $\mathrm{NO}_{3}$ plasma concentration prior to heme arginate administration as well as prior to and 15 minutes after ischemia as previously described [28].

Descriptive statistics were used for outcome and safety parameters. To detect a treatment effect in a continuous outcome parameter of one standard deviation by a paired $t$-test with a two-sided significance level of 5\%, 12 subjects had to be recruited to achieve a power of approximately $90 \%$. With a maximum of two subjects dropping out (16.7\%), power is still at $80 \%$. The fitted measures time to peak, peak BOLD signal and slope of reperfusion signal decline of each muscle of interest were analyzed as dependent variables using a general linear mixed model, including the fixed factors 'treatment day' (placebo or administration of heme arginate) and muscle (soleus, gastrocnemius and tibialis anterior), and the random factor 'subject'. An interaction of treatment day and muscle was evaluated and dropped from the model if not significant. Pairwise post-hoc comparisons between muscles were corrected for multiple testing using the Fisher's least significant difference procedure. A twosided p-value lower than 0.05 was considered indicating statistical significance. PASW 18.0 (SPSS Inc., Chicago, Illinois, USA) was used for statistical computations.

\section{Results}

HA was tolerated well without adverse reaction. No significant difference of vital signs or safety blood parameters could be detected between study periods (Table 1). An increased creatine kinase serum concentration of up to $490 \mathrm{U} / 1$ occurred 24 hours after ischemia in two different subjects (one receiving HA, one receiving placebo) but resolved spontaneously.

Heme arginate administration 24 hours prior to ischemia increased peak BOLD reperfusion signal from 104.5 $\pm 0.6 \%$ to $106.2 \pm 0.6 \%$ compared to placebo (Figure $2 \mathrm{~d}$; $\mathrm{p}=0.025)$. Furthermore, the time to peak reperfusion was reduced from $221 \pm 19$ seconds to $175 \pm 16$ seconds versus placebo $(p=0.012)$. The calculated slope of reperfusion signal decline after peak was significantly steeper after heme arginate compared to control condition $\left(-2.8^{*} 10^{-4} \pm 0.2 * 10^{-4}\right.$ vs. $\left.-2.1 * 10^{-4} \pm 0.2 * 10^{-4} ; \quad \mathrm{p}=0.002\right)$. There was no significant effect of heme arginate on the BOLD signal during ischemia.

Table 1 Vital signs in treatment periods with placebo or heme arginate

\begin{tabular}{lcc}
\hline & Placebo & $\begin{array}{c}\text { Heme } \\
\text { arginate }\end{array}$ \\
\hline Systolic blood pressure pre ischemia $(\mathrm{mmHg})$ & $115 \pm 10$ & $114 \pm 8$ \\
Diastolic blood pressure pre ischemia $(\mathrm{mmHg})$ & $80 \pm 8$ & $77 \pm 8$ \\
Heart rate pre ischemia (bpm) & $73 \pm 4$ & $72 \pm 4$ \\
Systolic blood pressure post ischemia (mmHg) & $116 \pm 10$ & $115 \pm 9$ \\
Diastolic blood pressure post ischemia (mmHg) & $81 \pm 7$ & $80 \pm 10$ \\
Heart rate post ischemia (bpm) & $71 \pm 2$ & $70 \pm 3$ \\
Blood iron ( $\mathrm{mg} / \mathrm{dl})$ & $110 \pm 41$ & $100 \pm 38$ \\
Protein (g/l) & $76.0 \pm 3.8$ & $75.9 \pm 2.3$ \\
Lactate dehydrogenase $(\mathrm{U} / \mathrm{l})$ & $152 \pm 28$ & $154 \pm 28$ \\
Creatine kinase $(\mathrm{U} / \mathrm{l})$ & $148 \pm 114$ & $146 \pm 87$ \\
C - reactive protein $(\mathrm{mg} / \mathrm{dl})$ & $0.06 \pm 0.05$ & $0.08 \pm 0.09$
\end{tabular}

Data are presented as mean $\pm S D, n=12$. 

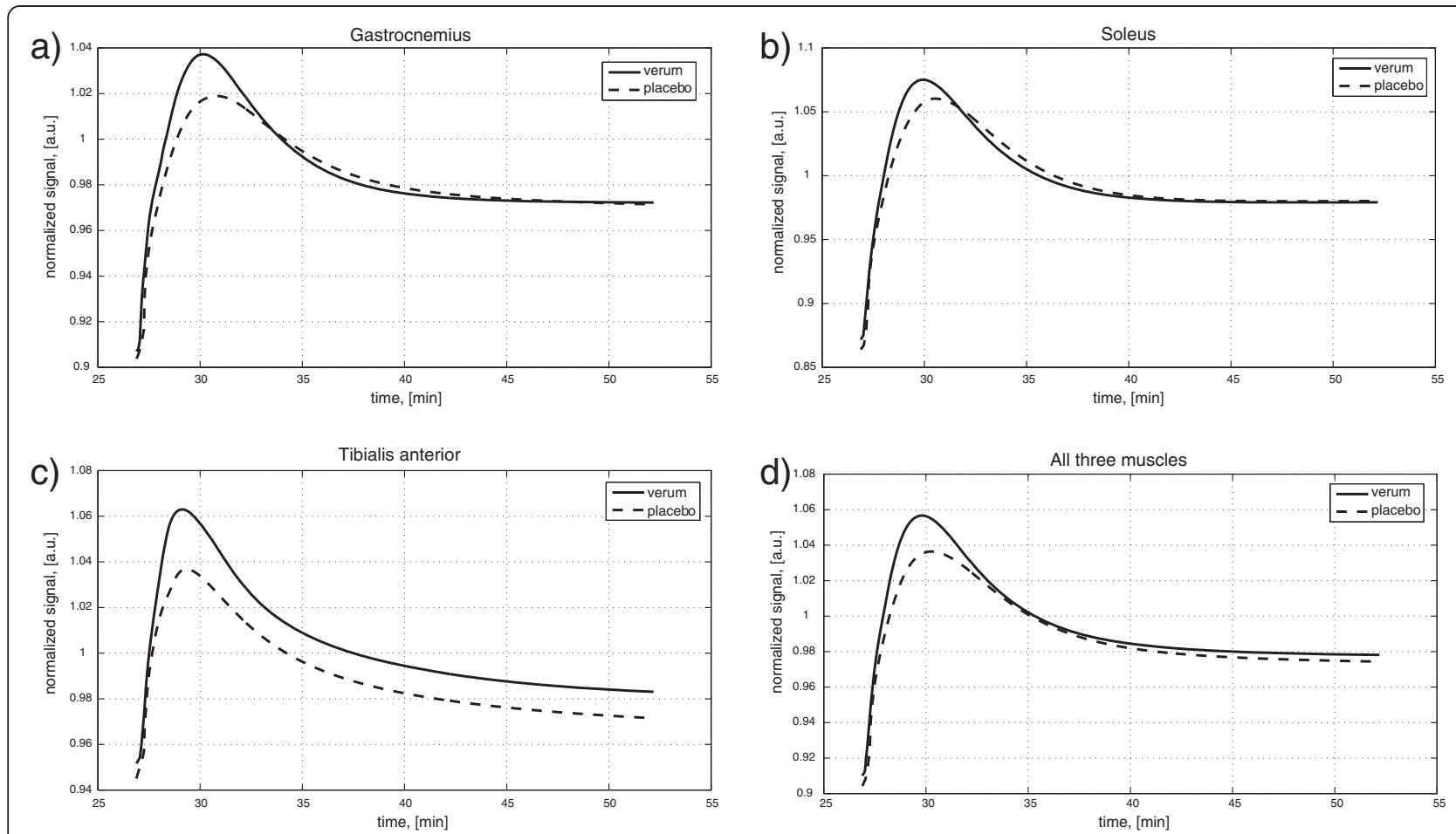

Figure 2 BOLD signal during reperfusion - group average showing treatment effect. a) gastrocnemius muscle; $\mathbf{b})$ soleus muscle; c) tibialis anterior muscle; $\mathbf{d}$ ) all three muscles. Caption: Reperfusion signal was fitted according to the described protocol; a.u.: arbitrary units, normalized to the pre-ischemic signal; min: minutes.

Reperfusion parameters for single muscle groups are depicted in Figure 2a-c and summarized in Table 2. Peak reperfusion signal after placebo was highest in the soleus muscle and lowest in the gastrocnemius muscle $(106.5$

Table 2 BOLD signal of calf muscles after ischemiareperfusion injury

\begin{tabular}{llll}
\hline All muscles & TTP (s) & PEAK (\%) & \multicolumn{1}{c}{ slope (1/s) } \\
\hline placebo & $221 \pm 19$ & $104.5 \pm 0.6$ & $-2.1^{*} 10^{-4} \pm 0.2^{*} 10^{-4}$ \\
HA & $175 \pm 16$ & $106.2 \pm 0.6$ & $-2.8^{*} 10^{-4} \pm 0.2^{*} 10^{-4}$ \\
p-value & 0.012 & 0.025 & 0.002 \\
\hline
\end{tabular}

\section{gastrocnemius}

\begin{tabular}{llll} 
placebo & $250 \pm 36$ & $102.6 \pm 0.8$ & $-1.6^{*} 10^{-4} \pm 0.2^{*} 10^{-4}$ \\
HA & $198 \pm 11$ & $104.0 \pm 0.6$ & $-2.2^{*} 10^{-4} \pm 0.2^{*} 10^{-4}$ \\
\hline
\end{tabular}

\section{soleus}

\begin{tabular}{llll} 
Placebo & $244 \pm 31$ & $106.5 \pm 0.7$ & $-2.6^{*} 10^{-4} \pm 0.4^{*} 10^{-4}$ \\
HA & $182 \pm 8$ & $107.8 \pm 0.8$ & $-3.2^{*} 10^{-4} \pm 0.4^{*} 10^{-4}$ \\
\hline
\end{tabular}

\section{Tibialis ant.}

$\begin{array}{llll}\text { Placebo } & 169 \pm 28 & 104.3 \pm 1.2 & -2.0^{*} 10^{-4} \pm 0.3^{*} 10^{-4} \\ \text { HA } & 152 \pm 13 & 106.9 \pm 1.5 & -3.0^{*} 10^{-4} \pm 0.4^{*} 10^{-4}\end{array}$

Heme arginate or placebo were administered 24 hours prior to ischemia. Data are mean $\pm S D, n=11 ; p$-value was calculated using a general linear mixed model and applies to all comparisons, since interaction analysis suggested homogeneity of the treatment effect across muscles. TTP: time to peak; PEAK: peak reperfusion signal; slope: slope of the tangent at the inflection point of the BOLD signal curve after the peak; $H A$ : heme arginate. $\pm 0.7 \%$ vs. $102.6 \pm 0.8 \%, \mathrm{p}=0.019$ ). A similar reaction across calf muscles to heme arginate could be confirmed by statistical analysis.

Plasma concentrations of $\mathrm{NO}_{2}$ and $\mathrm{NO}_{3}$ were 2.7 $\pm 1.4 \mu \mathrm{mol} / \mathrm{l}$ and $13.1 \pm 8.3 \mu \mathrm{mol} / \mathrm{l} 24$ hours after heme arginate administration compared to $3.5 \pm 1.8 \mu \mathrm{mol} / \mathrm{l}$ and $12.4 \pm 8.3 \mu \mathrm{mol} / \mathrm{l}$ after placebo, respectively $(\mathrm{p}=$ not significant). Plasma concentrations of $\mathrm{NO}_{2}$ and $\mathrm{NO}_{3}$ before heme arginate administration and after ischemia were also unchanged.

\section{Discussion}

This study demonstrates beneficial effects of HA pretreatment on endothelial function and reperfusion patterns after IRI in healthy humans. Full reperfusion signal occurred earlier, was higher and declined faster compared to placebo. We have previously reported that BOLD fMRI is able to characterize reperfusion patterns and the protective effects of ischemic preconditioning in IRI experiments [27]. Furthermore, we have shown the ability of HA to induce heme oxygenase- 1 mRNA and protein in humans [21]. The induction of HO-1 proved beneficial in several ischemia reperfusion models in animal studies $[11,17,19]$. However, the application of HA against IRI in humans has not been reported yet.

We propose a stronger and earlier peak BOLD signal during reperfusion as the healthy response. This 
hypothesis is supported by two prior trials showing a decreased BOLD fMRI signal in elderly subjects $[25,26]$. Furthermore, subjects with overt peripheral artery occlusive disease presented with decreased and delayed peak BOLD signal during reperfusion [24]. HA increased the peak reperfusion signal by $38 \%$ compared to the increase over baseline after placebo administration. This is in line with one report showing a $44 \%$ higher peak signal in healthy subjects compared to the BOLD signal increase during reperfusion in elderly subjects [26]. We could furthermore demonstrate a faster decline of the reactive hyperemia signal after the peak in the HA period, indicated by a steeper decline of the slope in the infliction point of the reperfusion signal curve.

The BOLD fMRI signal is determined by a combined influence of perfusion, vascular microarchitecture and oxygen saturation of hemoglobin and myoglobin. Unfortunately, the individual contributions of each of these factors to the finally observed signal cannot be separated. Regions of interest were drawn around muscles to exclude confounding signal influences from main vessels, fatty tissue and bones. The soleus muscle, a slowtwitch fibre muscle, is known to show a high capillary density [29]. It showed the highest peak reperfusion value, as also reported in previous trials [26,27]. Vasodilation of muscular capillaries may therefore be a strong contributor to the BOLD signal. Since this trial had a crossover design and results were analysed with a repeated measurement model, an influence of vessel architecture to the different peak signals is unlikely. Improved perfusion may therefore be the major contributor to the peak BOLD signal. However, muscular metabolism may also influence oxygen extraction and hemoglobin saturation during reperfusion [27]. Therefore, the BOLD signal cannot be directly compared to reperfusion patterns measured by flow-mediated dilation or strain gauge plethysmography [30]. It seems plausible that a protected endothelial cell function is responsible for faster and increased maximal reperfusion, leading also to more rapid and increased oxygen saturation in the muscles under study. The faster normalization of the reperfusion signal after peak may be an additional sign of protected endothelial cell function and adequate early reperfusion of muscular tissue. This protection may be mediated by decreased heme toxicity and increased antioxidative capacity due to $\mathrm{HO}-1$ induction and the action of its enzymatic products biliverdin/bilirubin and carbon monoxide [11]. Ischemia leads to a stasis of red blood cells in the ischemic tissue. Red blood cells are subjective to reactive oxygen radicals which are increasingly produced during ischemia and early reperfusion [31,32].

A limitation of this trial is the short period of ischemia. An increased ischemic period may reveal greater tissue injury and alter the protective effects of HA administration but is not feasible due to ethical concerns. Further, we have not measured HO-1 mRNA and protein levels in this trial. Clinical studies in patients with acute ischemic events are mandatory to validate these experiments accordingly. To draw more general conclusions, a female group and subjects with different underlying diseases should be included in future studies.

Arginine, the substrate of nitric oxide synthase, may exert systemic vasodilator effects mediated by nitric oxide [33]. To analyse a possible contribution of increased nitric oxide synthase activity induced by heme arginate, $\mathrm{NO}_{2}$ and $\mathrm{NO}_{3}$ levels were measured in plasma. The lack of changes in systemic nitric oxide metabolites argues against major vascular nitric oxide stimulation as a confounder of our results.

Our interpretation has to be discussed with caution in the context of previously published results. We reported an increased phosphocreatine overshoot and a decreased peak BOLD signal after ischemic preconditioning four hours prior to 20 minutes of ischemia. The phosphocreatine overshoot indicated an improved mitochondrial function by ischemic preconditioning, which may explain the increased oxygen demand leading to a decreased peak BOLD signal after ischemic preconditioning. Therefore, we suggest that the protecting effects of HA are different from the effects of ischemic preconditioning. The induction of HO-1 seems to be downstream in the cascade of ischemic preconditioning $[34,35]$. Therefore, different patterns in fMRI time series of these two protecting interventions as demonstrated by fMRI so far are reasonable. Furthermore, the fMRI measurement parameters were not identical in all studies. We increased the number of slices and decreased the slice gap for reduced artefacts from through-plane motion at the cost of reduced temporal resolution and shorter echo time. This enabled better signal-to-noise ratio but showed a slightly different contrast. Therefore, a direct, numerical comparison with our previous study is not possible [27].

\section{Conclusions}

In conclusion, application of heme arginate improves reperfusion patterns during IRI and may protect endothelial cells and skeletal muscle against IRI in humans. The potential clinical applications of this intervention have to be addressed in further trials.

\section{Competing interests}

The authors declare that they have no competing interests.

\section{Authors' contributions}

All authors fulfill the criteria for authorship. MA, DD and MW designed and drafted the interventional protocol, AIS, KS and EM developed and applied the fMRI protocols. MA and SW performed ischemia reperfusion experiments. $\mathrm{MB}$ and $\mathrm{GH}$ performed laboratory analysis and supported the calculation and 
interpretation of endpoint measures. MA and MW wrote the final draft. All authors have read and approved submission of the final draft.

\section{Acknowledgements}

This work has been funded by the Vienna Science and Technology Fund (WWTF) through project LS07-031.

\section{Author details \\ 'Department of Clinical Pharmacology, Medical University of Vienna, Vienna, Austria. ${ }^{2}$ Department of Cardiac Surgery, Medical University of Vienna, Vienna, Austria. ${ }^{3}$ MR Center of Excellence, Center for Biomedical Engineering and Physics, Medical University of Vienna, Vienna, Austria. ${ }^{4}$ Center for Medical Statistics, Informatics and Intelligent Systems, Medical University of Vienna, Vienna, Austria. ${ }^{5}$ Department of Laboratory Medicine, Medical University of Vienna, Vienna, Austria}

Received: 29 October 2011 Accepted: 9 July 2012

Published: 2 August 2012

\section{References}

1. Matsumura K, Jeremy RW, Schaper J, Becker LC: Progression of myocardial necrosis during reperfusion of ischemic myocardium. Circulation 1998, 97:795-804.

2. Eefting F, Rensing B, Wigman J, Pannekoek WJ, Liu WM, Cramer MJ, Lips DJ, Doevendans PA: Role of apoptosis in reperfusion injury. Cardiovasc Res 2004, 61:414-426.

3. Zweier $\mathrm{LL}$, Talukder MA: The role of oxidants and free radicals in reperfusion injury. Cardiovasc Res 2006, 70:181-190.

4. Vinten-Johansen J: Involvement of neutrophils in the pathogenesis of lethal myocardial reperfusion injury. Cardiovasc Res 2004, 61:481-497.

5. Zhao ZQ, Vinten-Johansen J: Postconditioning: reduction of reperfusioninduced injury. Cardiovasc Res 2006, 70:200-211.

6. Pleiner J, Schaller G, Mittermayer F, Bayerle-Eder M, Roden M, Wolzt M: FFAinduced endothelial dysfunction can be corrected by vitamin C. J Clin Endocrinol Metab 2002, 87:2913-2917.

7. Bayerle-Eder M, Pleiner J, Mittermayer F, Schaller G, Roden M, WaldhausI W, Bieglmayer C, Wolzt M: Effect of systemic vitamin C on free fatty acidinduced lipid peroxidation. Diabetes Metab 2004, 30:433-439.

8. Murry CE, Jennings RB, Reimer KA: Preconditioning with ischemia: a delay of lethal cell injury in ischemic myocardium. Circulation 1986, 74:1124-1136.

9. Kharbanda RK, Peters M, Walton B, Kattenhorn M, Mullen M, Klein N, Vallance P, Deanfield J, MacAllister R: Ischemic preconditioning prevents endothelial injury and systemic neutrophil activation during ischemiareperfusion in humans in vivo. Circulation 2001, 103:1624-1630.

10. Gori T, Dragoni S, Di Stolfo G, Sicuro S, Liuni A, Luca MC, Thomas G, Oelze M, Daiber A, Parker JD: Tolerance to nitroglycerin-induced preconditioning of the endothelium: a human in vivo study. Am J Physiol Heart Circ Physiol 2010, 298:H340-H345.

11. Ryter SW, Alam J, Choi AM: Heme oxygenase-1/carbon monoxide: from basic science to therapeutic applications. Physiol Rev 2006, 86:583-650.

12. Tenhunen R, Marver HS, Schmid R: The enzymatic conversion of heme to bilirubin by microsomal heme oxygenase. Proc Natl Acad Sci USA 1968, 61:748-755.

13. Vitek L, Schwertner HA: The heme catabolic pathway and its protective effects on oxidative stress-mediated diseases. Adv Clin Chem 2007, 43:1-57.

14. Terry CM, Clikeman JA, Hoidal JR, Callahan KS: TNF-alpha and IL-1alpha induce heme oxygenase-1 via protein kinase $\mathrm{C}, \mathrm{Ca} 2+$, and phospholipase A2 in endothelial cells. Am J Physiol 1999, 276:H1493-H1501.

15. Kawamura K, Ishikawa K, Wada Y, Kimura S, Matsumoto H, Kohro T, Itabe H, Kodama T, Maruyama Y: Bilirubin from heme oxygenase-1 attenuates vascular endothelial activation and dysfunction. Arterioscler Thromb Vasc Biol 2005, 25:155-160.

16. Otterbein LE, Soares MP, Yamashita K, Bach FH: Heme oxygenase-1: unleashing the protective properties of heme. Trends Immunol 2003, 24:449-455.

17. Ferenbach DA, Nkejabega NC, McKay J, Choudhary AK, Vernon MA, Beesley MF, Clay S, Conway BC, Marson LP, Kluth DC, Hughes J: The induction of macrophage hemeoxygenase-1 is protective during acute kidney injury in aging mice. Kidney Int 2011, 79:966-976.

18. Maeshima K, Takahashi T, Uehara K, Shimizu H, Omori E, Yokoyama M, Tani $T$, Akagi R, Morita K: Prevention of hemorrhagic shock-induced lung injury by heme arginate treatment in rats. Biochem Pharmacol 2005, 69:1667-1680.

19. Sasaki T, Takahashi T, Maeshima K, Shimizu H, Toda Y, Morimatsu H, Takeuchi M, Yokoyama M, Akagi R, Morita K: Heme arginate pretreatment attenuates pulmonary NF-kappaB and AP-1 activation induced by hemorrhagic shock via heme oxygenase-1 induction. Med Chem 2006 2:271-274.

20. Bharucha AE, Kulkarni A, Choi KM, Camilleri M, Lempke M, Brunn GJ, Gibbons SJ, Zinsmeister AR, Farrugia G: First-in-human study demonstrating pharmacological activation of heme oxygenase- 1 in humans. Clin Pharmacol Ther 2010, 87:187-190.

21. Doberer D, Haschemi A, Andreas M, Zapf TC, Clive B, Jeitler M, Heinzl H, Wagner $\mathrm{O}$, Wolzt $M$, Bilban M: Haem arginate infusion stimulates haem oxygenase-1 expression in healthy subjects. Br J Pharmacol 2010, 161:1751-1762.

22. Meyerspeer M, Kemp GJ, Mlynarik V, Krssak M, Szendroedi J, Nowotny P, Roden M, Moser E: Direct noninvasive quantification of lactate and high energy phosphates simultaneously in exercising human skeletal muscle by localized magnetic resonance spectroscopy. Magn Reson Med 2007, 57:654-660

23. Meyerspeer M, Krssak M, Kemp GJ, Roden M, Moser E: Dynamic interleaved $1 \mathrm{H} / 31 \mathrm{P}$ STEAM MRS at 3 Tesla using a pneumatic force-controlled plantar flexion exercise rig. MAGMA 2005, 18:257-262.

24. Ledermann HP, Schulte AC, Heidecker HG, Aschwanden M, Jager KA, Scheffler K, Steinbrich W, Bilecen D: Blood oxygenation level-dependent magnetic resonance imaging of the skeletal muscle in patients with peripheral arterial occlusive disease. Circulation 2006, 113:2929-2935.

25. Kos S, Klarhofer M, Aschwanden M, Scheffler K, Jacob AL, Bilecen D: Simultaneous dynamic blood oxygen level-dependent magnetic resonance imaging of foot and calf muscles: aging effects at ischemia and postocclusive hyperemia in healthy volunteers. Invest Radiol 2009, 44:741-747.

26. Schulte AC, Aschwanden M, Bilecen D: Calf muscles at blood oxygen level-dependent MR imaging: aging effects at postocclusive reactive hyperemia. Radiology 2008, 247:482-489.

27. Andreas M, Schmid Al, Keilani M, Doberer D, Bartko J, Crevenna R, Moser E, Wolzt M: Effect of ischemic preconditioning in skeletal muscle measured by functional magnetic resonance imaging and spectroscopy: a randomized crossover trial. J Cardiovasc Magn Reson 2011, 13:32.

28. Schmidt HH, Warner TD, Nakane M, Forstermann U, Murad F: Regulation and subcellular location of nitrogen oxide synthases in RAW264.7 macrophages. Mol Pharmacol 1992, 41:615-624.

29. Noseworthy MD, Bulte DP, Alfonsi J: BOLD magnetic resonance imaging of skeletal muscle. Semin Musculoskelet Radiol 2003, 7:307-315.

30. Utz W, Jordan J, Niendorf T, Stoffels M, Luft FC, Dietz R, Friedrich MG: Blood oxygen level-dependent MRI of tissue oxygenation: relation to endothelium-dependent and endothelium-independent blood flow changes. Arterioscler Thromb Vasc Biol 2005, 25:1408-1413.

31. Tsuchihashi S, Fondevila C, Kupiec-Weglinski JW: Heme oxygenase system in ischemia and reperfusion injury. Ann Transplant 2004, 9:84-87.

32. Wagener FA, Volk HD, Willis D, Abraham NG, Soares MP, Adema GJ, Figdor CG: Different faces of the heme-heme oxygenase system in inflammation. Pharmacol Rev 2003, 55:551-571.

33. Wolzt M, Schmetterer L, Ferber W, Artner E, Mensik C, Eichler HG, Krejcy K: Effect of nitric oxide synthase inhibition on renal hemodynamics in humans: reversal by L-arginine. Am J Physiol 1997, 272:F178-F182.

34. Franco-Gou R, Rosello-Catafau J, Casillas-Ramirez A, Massip-Salcedo M, Rimola A, Calvo N, Bartrons R, Peralta C: How ischaemic preconditioning protects small liver grafts. J Pathol 2006, 208:62-73.

35. Park MK, Kang YJ, Lee HS, Kim HJ, Seo HG, Lee JH, Chang KC: The obligatory role of COX-2 expression for induction of $\mathrm{HO}-1$ in ischemic preconditioned rat brain. Biochem Biophys Res Commun 2008, 377:1191-1194.

\section{doi:10.1186/1532-429X-14-55}

Cite this article as: Andreas et al.: Heme arginate improves reperfusion patterns after ischemia: a randomized, placebo-controlled trial in healthy male subjects. Journal of Cardiovascular Magnetic Resonance 2012 14:55. 